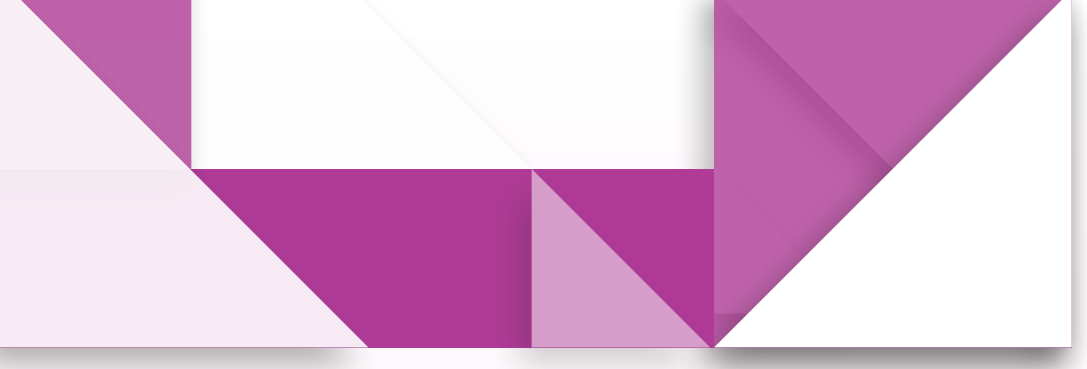

\title{
La enseñanza de caída libre 3 bajo la metodología de aprendizaje activo
}

\author{
- Teaching Free Fall under the Active Learning \\ Methodology \\ - ensino da queda livre sob a metodologia
da aprendizagem ativa
}

\section{Resumen}

En este artículo de investigación se exponen las conclusiones derivadas de la ejecución del proyecto "La enseñanza de caída libre bajo la metodología de aprendizaje activo (MAA)", el objetivo de este trabajo es presentar los resultados de la implementación de la unidad didáctica para la enseñanza de caída libre en grado décimo, diseñada bajo la MAA. La metodología orientadora del proyecto fue la investigación acción. Mediante el proceso cíclico en el que se sustenta dicha metodología, se planeó, ejecutó, analizó y evaluó la unidad y a partir de los resultados obtenidos se fueron haciendo los ajustes a su diseño. La propuesta fue implementada en el grado 10-1 de la Institución Educativa Emiliano Restrepo Echavarría del municipio de Restrepo (Meta, Colombia). Para analizar la efectividad de la metodología empleada se trabajó con la ganancia de aprendizaje o factor de Hake, encontrándose que a escala global se obtuvo una ganancia de aprendizaje conceptual medio, dado que los estudiantes logran relacionar para el movimiento de caída libre las variables tiempo y velocidad de caída, argumentando cómo a medida que un cuerpo cae gana velocidad y para cuerpos que son soltados desde la misma altura y de forma simultánea, el tiempo de caída es aproximadamente el mismo.

Palabras clave

aprendizaje activo; enseñanza de la física; unidad didáctica
Nasly Yanira Martínez Velásquez ${ }^{1}$ Sindy Yuley Riveros Míguez ${ }^{2}$

1 Magíster en Ciencias Físicas. Docente auxiliar de la Universidad de los Llanos, Facultad de Ciencias Humanas y de la Educación. Villavicencio, Colombia.

nmartinez@unillanos.edu.co

2 Licenciada en Matemáticas y Física. Universidad de los Llanos, Facultad de Ciencias Humanas y de la Educación. Villavicencio, Colombia. sindy.riveros@unillanos.edu.co 


\begin{abstract}
In this research paper we present the conclusions resulting from the project "Teaching free fall under the active learning methodology - ALM." The purpose of this work is to share the results of implementing the Didactic Unit for teaching free fall in the tenth grade designed under the ALM (Active Learning Methodology); the guiding methodology of the project was action research. The unit was planned, executed, analyzed and evaluated through the cyclic process in which this methodology is grounded, and adjustments were made to its design based on the results obtained. The proposal was implemented in class 10-1 of Institución Educativa Emiliano Restrepo Echavarría in the municipality of Restrepo (Meta, Colombia). In order to analyze the effectiveness of the methodology used, we worked with the learning gain or Hake factor, finding that, on a global scale level, a medium conceptual learning gain was obtained, given that students are able to relate the variables of time and speed of fall to the free fall movement, arguing how a body gains speed as it falls and how the time of fall is approximately the same for bodies that are released from the same height and at the same time.

Keywords

active learning; teaching physics; didactic unit

\section{Resumo}

Neste trabalho de pesquisa apresentamos as conclusões decorrentes da implementação do projeto "O ensino da queda livre sob a metodologia de aprendizado ativo-MAA -". O objetivo deste trabalho é apresentar os resultados da implementação da Unidade Didática para o ensino da queda livre na décima série, projetada sob o MAA (Metodologia de Aprendizagem Ativa); A metodologia orientadora do projeto foi a pesquisa-ação. Por meio do processo cíclico em que esta metodologia é sustentada, a unidade foi planejada, executada, analisada e avaliada e, com base nos resultados obtidos, foram realizados ajustes em seu desenho. A proposta foi implementada na décima série da Instituição de Ensino Emiliano Restrepo Echavarría, no município de Restrepo (Meta,-Colômbia). Para analisar a eficácia da metodologia trabalhamos com um ganho de aprendizagem ou fator de Hake, descobrindo que o ganho de aprendizagem a escala global foi conceitual médio, já que os alunos são capazes de se relacionar o movimento de queda livre com as variáveis tempo e velocidade de queda, argumentando como um corpo ganha velocidade enquanto cai e como o tempo de queda é aproximadamente o mesmo para corpos que são liberados desde a mesma altura e simultaneamente

Palavras-chave

aprendizagem ativa; ensino de física; unidade didática
\end{abstract}




\section{Introducción}

Para McDermott (2001) la enseñanza tradicional (ET), como se ha demostrado en diversos estudios, no permite que el estudiante realice adecuadas conexiones entre conceptos, representaciones formales y situaciones del mundo real. Las concepciones previas, en algunos casos, erradas, no son superadas bajo este esquema de enseñanza, no se promueve una estructura conceptual coherente y no se incrementa en capacidad de análisis y razonamiento. La responsabilidad del proceso de enseñanza-aprendizaje recae en el docente, el estudiante no es el centro de este, es un agente pasivo y receptor, la autoridad intelectual la tienen el profesor y los libros de texto y debido al afán por cumplir con un plan curricular, no hay tiempo para escenarios en los que se genere gusto y actitud por el asombro, por la observación, por la pregunta, por la conjetura, por el análisis, como señalan Bruner (1987), Barrera (2007), citados por Barbosa (2008), generando en los estudiantes - como afirman Solbes, Monserrat y Furio (1997)_, una valoración negativa de las ciencias, que las hacen difíciles, aburridas, con currículos rígidos y en donde la falta de conexión entre los contenidos y la vida diaria hace que les resulten poco atractivas, confirmando el bajo interés y motivación por su estudio (Villarreal et al., 2005).

Además de lo anterior, el deficiente desarrollo de habilidades comunicativas propias de las temáticas requeridas para la física, el ignorar que los estudiantes tienen experiencias previas del mundo real y que las tienen organizadas de una forma particular que les explique, a su modo, los hechos reales (Elizondo, 2013), ocasiona dificultades en el aprendizaje de dicha ciencia, convirtiéndose en un obstáculo para el avance en la comprensión de los principios y leyes en las que se sustenta y permiten explicar el comportamiento de la naturaleza.

Por otra parte, el trabajo de experimentos aislados y descontextualizados, la estructura de contenidos de manera rígida sin tener en cuenta el nivel de desarrollo de los estudiantes, la enseñanza memorística y la desarticulación de conceptos científicos con las ideas previas de los estudiantes, así como la estructura lógica de los contenidos conceptuales, el nivel de exigencia formal y la influencia de los conocimientos previos y preconcepciones del alumno, como lo señalan Campanario y Moya (1999), son poco eficaces para promover aprendizajes significativos.

Como estrategia alternativa a la enseñanza tradicional se propone la metodología de aprendizaje activo (MAA); bajo este enfoque los alumnos deben plantear sus predicciones acerca de diversas experiencias, argumentar las razones en las que se basan para formular las predicciones, realizar el diseño experimental, para, después, contrastar los resultados de la experiencia con sus predicciones iniciales, de esta manera se ayuda a los estudiantes a ser conscientes de sus propios procesos cognitivos; así mismo, con este tipo de tareas se llama la atención sobre el papel de la observación y validación de hipótesis en la ciencia.

\section{Fundamentación de la experiencia}

El aprendizaje activo se define como un método de instrucción que involucra a los estudiantes en el proceso de aprendizaje; se requiere que los estudiantes realicen actividades de aprendizaje y reflexionen acerca de lo que están haciendo, mientras que el papel del docente se visualiza como el generador de un clima de desequilibrio conceptual. El uso de técnicas activas logra un ambiente de aprendizaje 
más eficaz; al respecto Thornton y Sokoloff (1997) afirman que este permite el desarrollo de habilidades del pensamiento, es ágil, divertido, útil, lográndose con frecuencia que los alumnos abandonen sus asientos para moverse por el aula y pensar en voz alta (Silberman, 1998).

Con la metodología de aprendizaje activo (MAA) se busca generar un cambio procedimental y de protagonismo en el papel que desempeña tanto el docente, como el estudiante en los procesos educativos, en los que el profesor sea una guía del proceso de aprendizaje permitiendo que el estudiante realice tareas experimentales de observación, predicción, medición, análisis, contrastación y comparación, que propicien una mejor construcción de su conocimiento. Se busca en este enfoque, además, que se estimule la colaboración entre estudiantes en un ámbito de enseñanza orientado desde el trabajo cooperativo (Benegas, 2007; Benegas et al., 2011 ; McDermott, 2001, citados por Zapata et al., 2012).

En este orden de ideas, para que el proceso de enseñanza-aprendizaje de la física esté enmarcado en el tipo de metodología descrito, se requiere además de lo anterior, que se incluyan preguntas o situaciones que impliquen un razonamiento cualitativo y explicaciones verbales; se deben realizar prácticas sostenidas para interpretar el formalismo físico y relacionarlo con el mundo real, donde se articulen los conocimientos teóricos y prácticos (Franco, Velasco y Riveros, 2017). Los estudiantes deben participar en el proceso de construcción de modelos cualitativos y en la aplicación de estos para predecir y explicar los fenómenos observados, también, se debe cultivar el razonamiento científico (McDermott, 1993, citado por Benegas, 2007).

La MAA propuesta por Thornton y Sokoloff (1997), contempla ocho pasos para su implementación en el aula, los cuales están descritos en la figura 1. Para la estrategia en el aula existen dos variantes que pueden ayudar en su realización, clases interactivas demostrativas (CID) o laboratorios de aprendizaje activo (LAA) (Sokoloff y Thornton, 2004).

\section{Sistematización y análisis de la experiencia}

Para el desarrollo de la experiencia, en primer lugar, se planeó la unidad didáctica (UD); para ello se tuvo en cuenta la malla curricular de la Institución Educativa Emiliano Restrepo Echavarría, institución de carácter oficial del municipio de Restrepo (Meta). Se trabajó con una muestra de 39 estudiantes de grado 10-1 (año lectivo 2016), se diseñó para ser ejecutada en cuatro intervenciones; posteriormente, se implementó la UD, de manera simultánea se hizo la observación y la reflexión, de tal forma que si era preciso realizar ajustes al diseño de la UD para lograr el propósito, se hicieran. En la tabla 1 se presentan las situaciones propuestas que permitieron abordar la temática de caída libre. 


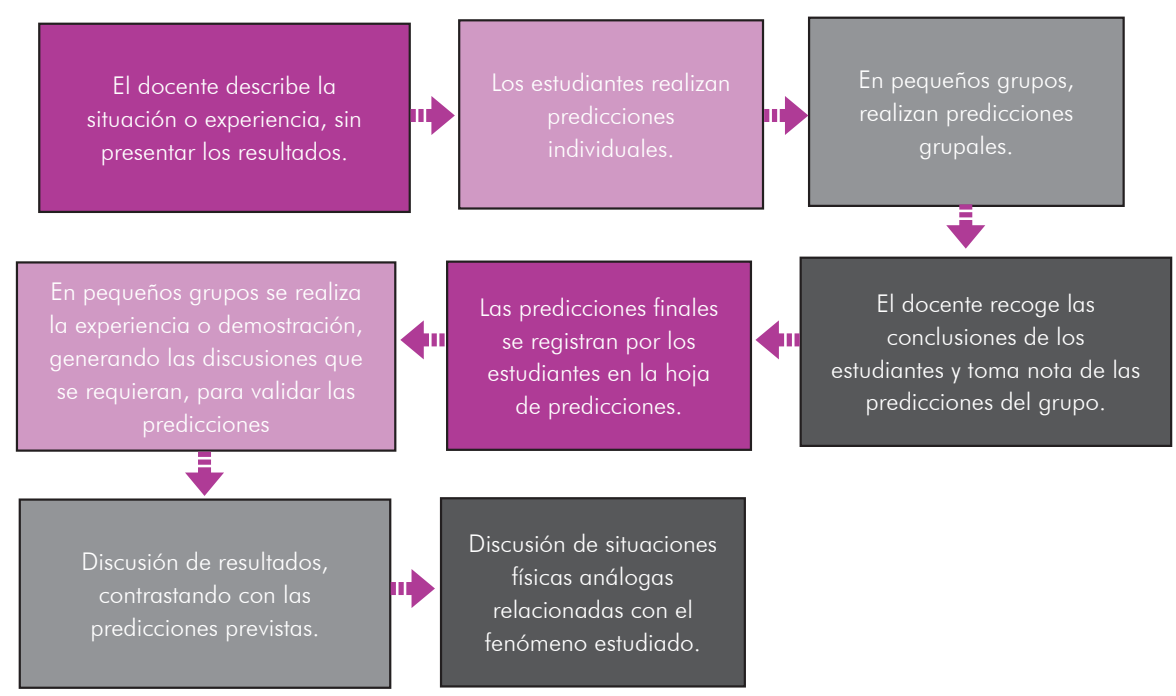

Figura 1. Pasos de la MAA

Fuente: adaptado de Sokoloff y Thornton (2004).

Tabla 1. Consolidado de situaciones propuestas en la UD para el desarrollo de la temática

\begin{tabular}{|c|c|c|}
\hline Secuencia & Actividades & Situación experimental o actividad propuesta \\
\hline Primera & $\begin{array}{l}\text { Prueba inicial } \\
\text { Planteamiento } \\
\text { de la situación } \\
\text { problema }\end{array}$ & $\begin{array}{l}\text { Se entrega la prueba de entrada (anexo 1) para que sea contestada de forma individual, } \\
\text { consta de nueve preguntas; las dos primeras son tomadas del cuestionario sobre el concepto } \\
\text { de fuerza (Hestenes, Wells y Swackhamer, 1992), las preguntas restantes se tomaron del } \\
\text { trabajo de Garduño, López y Mora (2013), validadas por los autores mencionados; con ella } \\
\text { se pretende identificar los conceptos previos que tienen los estudiantes frente a la caída de } \\
\text { los cuerpos, para analizar si su concepción está asociada a que caen más rápido los que son } \\
\text { más pesados, como lo afirman Gil y Valdés (1996): "Muchos de los estudiantes piensan que } \\
\text { mientras mayor sea la masa del cuerpo más rápidamente llegará al suelo". } \\
\text { En clase de física se suscita una discusión entre Juan y Claudia, dos estudiantes de grado } \\
\text { décimo, Juan afirma que si se dejan caer dos cuerpos desde la misma altura, por ejemplo, } \\
\text { una esfera de acero y una de plástico ', llega al suelo primero la esfera de acero que la de } \\
\text { plástico, porque tiene más masa. Por su parte, Claudia afirma que llegarán al mismo tiempo, } \\
\text { porque la caída de los cuerpos es independiente de la masa. ¿Con cuál compañero está de } \\
\text { acuerdo y por qué? }\end{array}$ \\
\hline Segunda & $\begin{array}{l}\text { Planteamiento } \\
\text { de la situación } \\
\text { problema }\end{array}$ & $\begin{array}{l}\text { Se tiene un zapato y una moneda, se dejan caer desde la misma altura y al mismo tiempo } \\
\text { sobre una lata de forma rectangular, żqué sucede con los objetos que caen? ¿̇Por qué? }\end{array}$ \\
\hline Tercera & $\begin{array}{l}\text { Situación } \\
\text { problema }\end{array}$ & $\begin{array}{l}\text { Se tiene una botella de plástico, la botella no contiene ningún líquido, se tapona la boca de } \\
\text { la botella con plastilina y se pone pitillo dentro de esta sostenido por la plastilina. } \\
\text { Luego se pone otro pitillo de mayor diámetro por encima del pitillo que está en la botella, se } \\
\text { debe tener precaución de haber tapado la parte de arriba de este pitillo. } \\
\text { ¿Qué sucede si se oprime la botella, estando ésta en sentido vertical? }{ }^{2}\end{array}$ \\
\hline Cuarta & Prueba final & Se realiza la prueba postest (anexo 1) de forma individual. \\
\hline
\end{tabular}

Fuente: Riveros (2016).

1 Manuel Francisco Alonso Sánchez (profesor), Ana Isabel Aracil Belmonte, Asier Martínez Ferrándiz, María Ortega Martínez y Antonio Rodríguez Ávila (alumnos de bachillerato), del IEs Leonardo da Vinci Alicante. Demostraciones experimentales sobre caída libre. Experiencias prácticas AA. W.: IES Leonardo da Vinci Alicante.

2 Disponible en https://www.youtube.com/watch? $\mathrm{v}=\mathrm{NZ8yGmAHJ} 4$ (citado el 15 de marzo del 2016). 
Para evaluar la efectividad de la estrategia, se trabajó con el factor de Hake (Hake, 1998) o ganancia relativa de aprendizaje conceptual, esta cantidad indica la ganancia promedio del aprendizaje conceptual y permite comparar el grado de logro de la estrategia educativa, en la ecuación 1 se presenta cómo se calcula el factor de Hake:

$$
h=\frac{\text { postest }(\%)-\text { Pretest }(\%)}{100-\text { Pretest }(100 \%)}
$$

Una ganancia de Hake baja se encuentra cuando $h<0,3$, media cuando $0,7>h \geq 0,30$ y alta para $h \geq 0,7$.

En la tabla 2 se presentan los resultados obtenidos en la aplicación del pretest, en donde se puede ver el porcentaje de estudiantes que seleccionaron cada una de las cinco opciones de respuesta para las nueve preguntas. El porcentaje de la respuesta correcta está señalado en negrita. Los datos corresponden al pretest de los 39 estudiantes de grado 10-1.

Tabla 2. Resultados del pretest

\begin{tabular}{|c|c|c|c|c|c|c|c|c|c|}
\hline \multirow[b]{2}{*}{$\begin{array}{l}\text { Respuesta } \\
\text { elegida }\end{array}$} & \multicolumn{9}{|c|}{ Pregunta } \\
\hline & P1 & P2 & P3 & P4 & P5 & P6 & P7 & P8 & P9 \\
\hline A & 28,21 & 23,08 & 12,82 & 25,64 & 46,15 & 15,38 & 51,28 & 17,95 & 35,90 \\
\hline B & 0,00 & 12,82 & 41,03 & 33,33 & 33,33 & 30,77 & 12,82 & 12,82 & 23,08 \\
\hline C & 30,77 & 12,82 & 28,21 & 23,08 & 10,26 & 35,90 & 15,38 & 20,51 & 5,13 \\
\hline D & 25,64 & 41,03 & 10,26 & 17,95 & 10,26 & 12,82 & 12,82 & 35,90 & 25,64 \\
\hline E & 15,38 & 10,26 & 7,69 & 0,00 & 0,00 & 5,13 & 7,69 & 12,82 & 10,26 \\
\hline
\end{tabular}

Fuente: elaboración propia.

En la figura 2, en el eje de las abscisas se grafican las nueve preguntas realizadas a los estudiantes en cada una de las cinco opciones de respuesta y en el eje de las ordenadas el porcentaje obtenido por los estudiantes en cada una de ellas. La opción de respuesta correcta está señalada con un asterisco amarillo.

En el ítem 1 se indaga por el tiempo de caída de dos esferas de igual tamaño y diferente masa que se dejan caer a la misma altura. Se encuentra que el 30,77\% de los estudiantes contestó correctamente la pregunta, opción C, afirmando que el tiempo de caída era igual para ambas esferas. Se observa que la opción de respuesta $A$ y $D$ es seleccionada por los estudiantes con porcentajes de $28,21 \%$ 
y $25,64 \%$, estas opciones de respuestas hacen énfasis en que la esfera de mayor peso se demora menos tiempo en caer, mientras que la opción E con un porcentaje de 15,38\%, señala que la esfera liviana llegaba primero al suelo. La opción B no fue considerada como respuesta entre los estudiantes, indicaba que la esfera más liviana tardaría aproximadamente la mitad del tiempo que la esfera pesada en llegar al suelo.

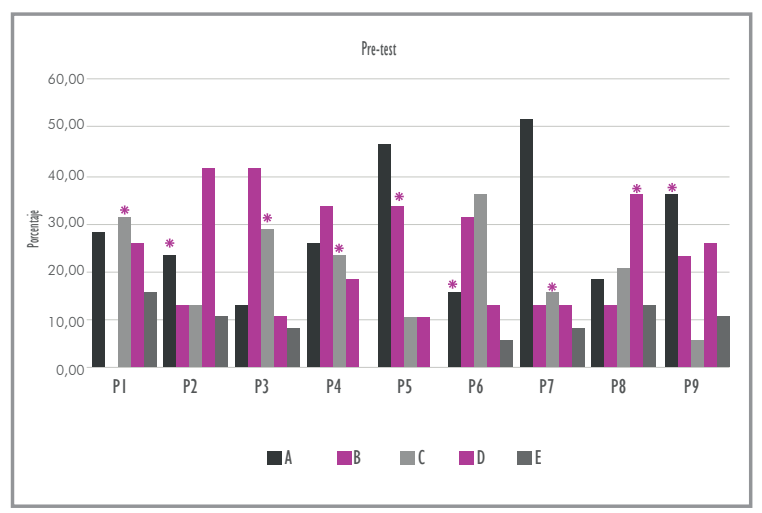

Figura 2. Resultados del pretest

Fuente: elaboración propia.

La pregunta 2 cuestiona sobre lo que pasaría si dos esferas de metal de igual tamaño y diferente peso ruedan sobre una mesa horizontal con la misma velocidad y caen al suelo al llegar al borde de la mesa. Un 23,08 \% de los estudiantes contestaron correctamente la opción A, considerando que ambas golpean el suelo aproximadamente a la misma distancia horizontal de la base de la mesa, mientras que la opción $D$ representada por el mayor porcentaje de selección de 41,03\% indicaba que la más pesada golpeaba el suelo considerablemente más cerca de la base de la mesa que la liviana. Para las opciones B y $\mathrm{C}$ el porcentaje fue menor, de 12,82\% cada una, la opción $A$ indicaba que la más pesada golpea el suelo aproximadamente a la mitad de la distancia horizontal de la base de la mesa que la más liviana, mientras que la opción $B$ decía lo contrario, y por último la opción $C$, seleccionada por un 10,26\% de los estudiantes, afirma que la más pesada golpea al suelo mucho más cerca que la liviana.

El ítem 3 pregunta por lo que sucede con la velocidad y la aceleración cuando se deja caer una piedra desde el techo de un edificio de un solo piso hasta la superficie de la tierra. Un 28,21\% de los estudiantes eligieron la respuesta correcta (opción C) que afirmaba que la velocidad de la piedra aumenta debido a una fuerza de gravedad constante que actúa sobre ella. Las opciones $\mathrm{A}$, D y $\mathrm{E}$ muestran porcentajes bajos de $12,82 \%, 10,26 \%$ y $7,69 \%$ respectivamente, la opción $A$ indica que alcanza un máximo de velocidad apenas se suelta la piedra y luego sigue su trayectoria con velocidad constante; en la opción $D$ se afirma que cae, debido a la tendencia natural de todos los cuerpos a descansar sobre la superficie y en la opción E cae debido a los efectos combinados de la fuerza de gravedad empujándola hacia abajo y la fuerza del aire empuiándola hacia arriba. La opción de respuesta $B$ resulta la opción incorrecta más popular entre los estudiantes con un porcentaje de $41,03 \%$, porque consideran que la velocidad de la piedra aumenta mientras cae debido a que la atracción gravitatoria se hace mayor cuanto más se acerca la piedra a la tierra.

En la pregunta 4, se quiere saber qué sucede con la velocidad inicial de una cubeta que se deja caer desde la azotea de un edificio, en donde un $23,08 \%$ de los estudiantes contestó correctamente la pregunta (opción C), considerando que su velocidad inicial era cero por partir del reposo, mientras que las opciones de respuesta $A$ y $B$ resultan ser opciones incorrectas y atractivas para los estudiantes con porcentajes del 25,64\% y $33,33 \%$ respectivamente. Con la opción A 
establecieron que al ser soltada la cubeta esta alcanza un máximo de velocidad y luego se mantiene con velocidad constante, y con la opción B que la velocidad aumentaba debido a la atracción gravitatoria que se hace mayor cuanto más se acerca a la tierra. En la opción D un 17,95\% de los estudiantes sugieren que la velocidad inicial aumenta aproximadamente a la mitad de su trayectoria. La opción E no fue considerada como respuesta entre los estudiantes, seguramente porque en esta opción se plantea que la velocidad inicial disminuye.

En el interrogante 5, se cuestiona sobre tiempo de caída de una moneda y una hoja de papel extendida que se dejan caer a la misma altura y en el mismo instante. El 33,33\% de los estudiantes contestó correctamente la opción B, en donde sugiere que el tiempo para la moneda será menor comparada con la hoja de papel extendida. La respuesta A fue la opción incorrecta con mayor porcentaje de selección con un 46,15\%; los estudiantes creen que el tiempo de caída es mayor para la moneda comparada con la hoja de papel. Las opciones C y D muestran porcentajes bajos de 10,26\% cada una, en la opción C el tiempo de caída es igual para ambos objetos, y en la opción D es aproximadamente la mitad para la moneda comparada con la hoja de papel extendida. La opción E no fue considerada por los estudiantes como opción de respuesta.

El numeral 6 averigua cómo es la aceleración de una pelota de ping-pong que se deja caer desde un edificio de dos pisos. Un 15,38\% de los estudiantes contestó correctamente al elegir la opción A, afirmando que su aceleración es constante. Se puede apreciar que las respuestas B y $C$ son opciones incorrectas escogidas por los estudiantes con porcentajes de $30,77 \%$ y $35,77 \%$ respectivamente, en la opción B se afirma que la aceleración aumenta debido a que la atracción gravitatoria se hace mayor cuanto más se acerca la pelota de ping-pong a la superficie y en la opción $C$ se señala que la aceleración aumenta porque la fuerza de gravedad es constante. Las opciones D y E muestran porcentajes bajos de selección, de 12,82\% y 5,13\% respectivamente, en estas opciones se considera que la aceleración disminuye debido a la tendencia natural de todos los cuerpos al caer o a la fuerza de gravedad que actúa sobre ella.

Para los ítems 7, 8 y 9, la situación que se propone es dejar caer una moneda verticalmente desde dos alturas distintas (altura mayor $\mathrm{H}$, altura menor h). En el 7 se interroga sobre la aceleración de la moneda justo al final de su recorrido. Tan solo un 15,38\% de los estudiantes contestó correctamente (opción C), indicando que la aceleración es igual para la moneda desde las dos alturas. La opción D, aunque es una respuesta incorrecta, es escogida por un buen número de estudiantes, con un $51,38 \%$ de elección, en ella se afirma que es mayor la aceleración si cae desde una altura $\mathrm{H}$. Las opciones $B$ y $D$ resultan ser muy poco consideradas por los estudiantes, logrando porcentajes de 12,82\% cada una, en ellas se afirma que la aceleración es menor si cae desde la altura $\mathrm{H}$ o justamente la mitad de la altura $\mathrm{H}$ comparado con el cuerpo que cae desde h. El 7,69\% consideró que ninguna de las opciones anteriores era la respuesta correcta y seleccionó la opción E. 
En el 8 se pregunta por el tiempo que tarda la moneda en llegar al suelo desde las alturas $\mathrm{H}$ y $\mathrm{h}$. Se encuentra que el 35,90\% de los estudiantes contestó correctamente la pregunta, opción $D$, indicando que es mayor el tiempo de caída si cae desde la altura $\mathrm{H}$. Para las opciones $\mathrm{A}$ y $\mathrm{C}$ se tiene un menor porcentaje de elección para cada una $(17,95 \%$ y $20,51 \%)$, en la opción A se menciona que el tiempo es mayor si la moneda cae desde la altura $h$ y en la opción $C$ que los tiempos son iguales para las alturas $\mathrm{H}$ y $\mathrm{h}$. Se observa que las opciones de respuesta $B$ y $D$ resultan poco interesantes para los estudiantes con igual porcentaje de 12,82\% cada una, la opción B sugiere que el tiempo es mayor si la moneda cae de la altura h y la $\mathrm{D}$ considera que ninguna de las anteriores respuestas es válida.

En el 9, el 35,90\% de los estudiantes contestó correctamente al elegir la opción A, indicando que, al caer, la moneda adquiere una velocidad distinta en cada punto de su trayectoria. También se puede apreciar que las respuestas $B$ y $D$ son las opciones que más escogen los estudiantes, con porcentajes del $23,08 \%$ y $25,64 \%$ respectivamente, la opción B afirma que la velocidad permanece constante y la opción $D$ que la velocidad es igual a la aceleración. La opción C muestra que un 5,13\% de los estudiantes consideran que la velocidad se comporta inversamente proporcional a la aceleración y la opción E que ninguna de las respuestas anteriores es la correcta.

Posterior a la aplicación del pretest, se realiza el análisis de las situaciones descritas en la tabla 1. En cada una de las intervenciones se realizó la secuencia de los ocho pasos presentados en la figura 1. En la figura 3 se muestra un ejemplo de las predicciones individuales relacionadas con la situación propuesta en la primera secuencia, en ella se observa que los estudiantes tienen una concepción aristotélica del movimiento de los cuerpos en caída libre.

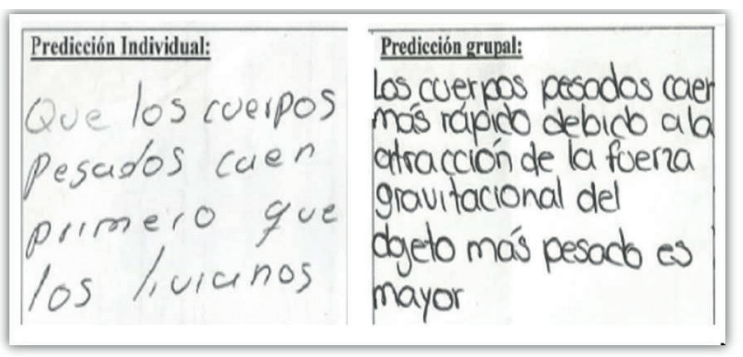

Figura 3. ¿̇Qué cae más rápido, la esfera de acero o la de plástico?

Fuente: elaboración propia.

En la figura 4 se presenta un estudiante realizando la demostración o diseño experimental; trabajaron en pequeños grupos, los mismos que se conformaron para las predicciones grupales, realizaron la práctica tantas veces como fue necesario, obteniendo sus propios resultados, para comparar luego con las predicciones y argumentar sus hallazgos.

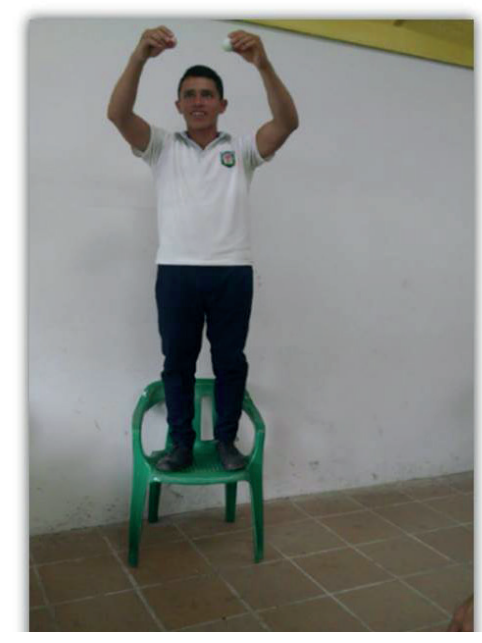

Figura 4. Demostración de esferas en caída libre Fuente: eelaboración propia.

En la figura 5 se presenta un ejemplo de las predicciones de los estudiantes frente a la situación de la secuencia dos, en la que se observa que los estudiantes van modificando sus concepciones previas asociadas con la caída de los cuerpos. 


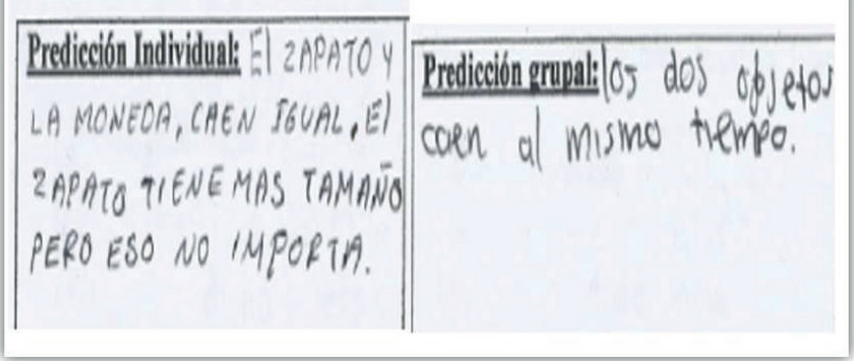

Figura 5. ¿̇Qué cae más rápido: un zapato o una moneda?

Fuente: elaboración propia.

En la figura 6 se evidencia el trabajo de LAA, de tal forma que los estudiantes pueden validar sus hipótesis y confrontar sus resultados.

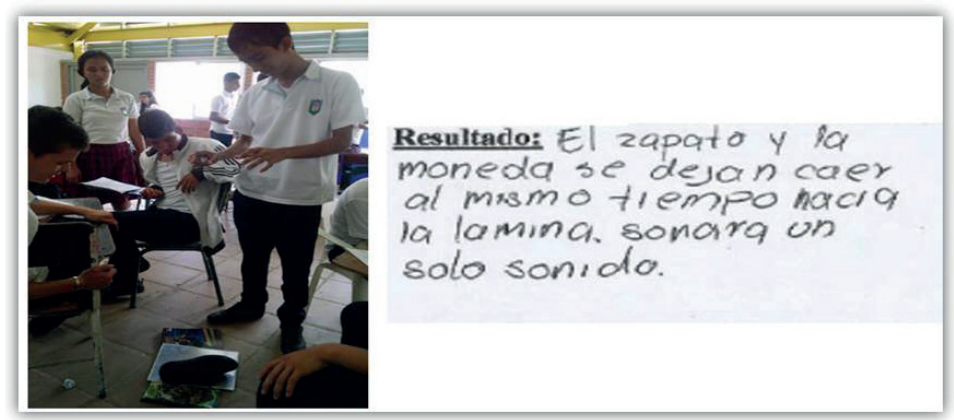

Figura 6. Demostración y resultado de caída de un zapato y una moneda

Fuente: elaboración propia.

En la figura 7 se presenta un ejemplo de las predicciones individuales relacionadas con la situación planteada en la tercera secuencia; los estudiantes predicen lo que consideran que va a suceder, sin embargo, no hablan de movimiento vertical hacia arriba, ni de cambios en la velocidad en función del ascenso del pitillo.

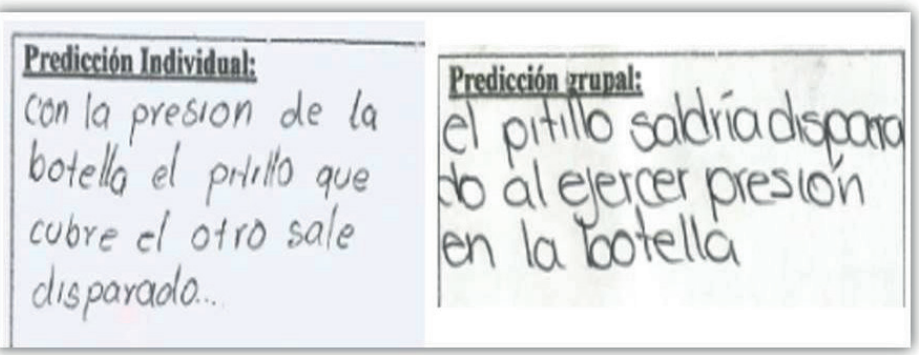

Figura 7. Predicciones del experimento de la botella Fuente: elaboración propia. 
En la figura 8 se observa a un estudiante realizando la práctica, posterior a la discusión de resultados, los estudiantes afirmaron que el pitillo sale disparado con una velocidad inicial y, como la botella está en dirección vertical, el pitillo se mueve verticalmente hacia arriba, la velocidad del pitillo va disminuyendo a medida que asciende, hasta que se hace cero en la parte más alta de su trayectoria.

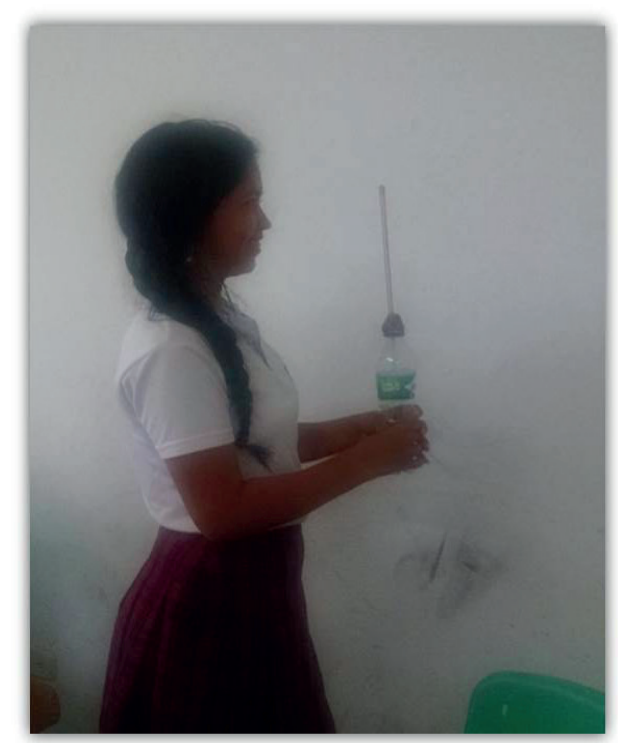

Figura 8. Demostración del experimento de la botella

Fuente: elaboración propia.

Finalmente se aplica el postest para evaluar qué sucedió con las ideas previas de los estudiantes luego de las intervenciones; en la tabla 3 se presentan los porcentajes de estudiantes que seleccionaron cada una de las cinco opciones de respuesta para las nueve preguntas de la prueba. El porcentaje de la respuesta correcta está señalado en negrita.
Tabla 3. Resultado del postest

\begin{tabular}{|c|c|c|c|c|c|c|c|c|c|}
\hline \multirow{2}{*}{ 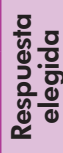 } & \multicolumn{9}{|c|}{ Pregunta } \\
\hline & P1 & P2 & P3 & P4 & 5 & P6 & P7 & P8 & P9 \\
\hline A & 7,69 & 69,23 & 38,46 & 10,26 & 38,46 & 56,41 & 38,46 & 2,56 & 61,54 \\
\hline B & 2,56 & 17,95 & 33,33 & 15,38 & 28,21 & 23,08 & 5,13 & 5,13 & 20,5 \\
\hline C & 87,18 & 2,56 & 20,51 & 71,79 & 25,64 & 7,69 & 43,59 & 7,69 & 5,13 \\
\hline D & 2,56 & 10,26 & 0,00 & 2,56 & 2,56 & 12,82 & 10,26 & 82,05 & 10,26 \\
\hline$E$ & 0,00 & 0,00 & 7,69 & 0,00 & 5,13 & 0,00 & 2,56 & 2,56 & 2,56 \\
\hline
\end{tabular}

Fuente: resultados de la prueba postest aplicada a los estudiantes de grado 10-1, de la Institución Educativa Emiliano Restrepo.

En figura 9 se presentan los resultados relacionados en la tabla anterior, se hace de forma similar a la figura 2 .

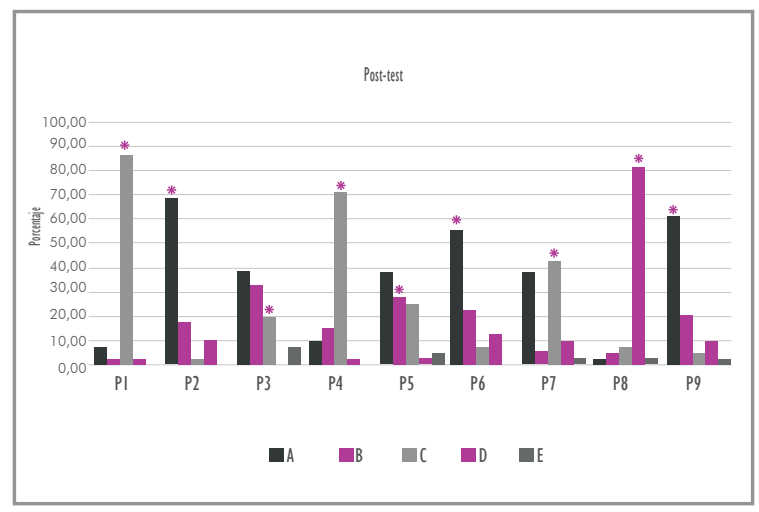

Figura 9. Resultados del postest

Fuente: elaboración propia.

Pregunta 1. Se pregunta por el tiempo que tardan dos esferas del mismo tamaño y diferente masa en llegar al suelo, cuando se dejan caer desde el techo de un edificio en el mismo instante. Un 87,18\% de los estudiantes contestó correctamente la pregunta con la opción C, afirmando que el tiempo empleado por las dos esferas al caer era aproximadamente el mismo. Para las opciones A, B y D muestran porcentajes inferiores al 7,69\%; la opción E no fue considerada como respuesta entre los estudiantes. En estas opciones se considera que los tiempos de caída varían de acuerdo con el peso de las esferas. 
Ítem 2. Si las dos esferas de metal del problema anterior ruedan sobre la mesa horizontal con la misma velocidad y caen al suelo al llegar al borde de la mesa. Ante esta situación, un 69,23\% de los estudiantes contestaron afirmativamente la opción $A$, indicando que ambas golpearían el suelo aproximadamente a la mitad de la distancia horizontal de la base. Sin embargo, las opciones B y $D$ muestran que un $17,95 \%$ y $10,26 \%$ de los estudiantes consideraron que la pesada golpea el suelo aproximadamente a la mitad o aún más cerca de la distancia horizontal de la base de la mesa que la bola liviana. La opción C y E no fue considerada como respuesta entre los estudiantes

Numeral 3. Cuando se pregunta sobre la velocidad que adquiere una piedra al dejarla caer desde el techo de un edificio de un solo piso hasta la superficie de la tierra. Solo el 20,51\% de los estudiantes eligieron afirmativamente la opción $\mathrm{C}$, indicando que la velocidad aumenta debido a una fuerza de gravedad constante que actúa sobre la piedra. Mientras que las respuestas A y B resultan ser opciones incorrectas con porcentajes del $38,46 \%$ y 33,33\%, superando a la respuesta correcta, los estudiantes consideran que efectivamente existe un cambio en la velocidad mientras cae la piedra, pero no fue clara la causa que generó ese aumento, asumiendo que la atracción gravitatoria se hace mayor a medida que se acerca a la superficie de la tierra. La opción D muestra porcentajes bajos de 7,69\% y la opción E no fue considerada como respuesta entre los estudiantes

Interrogante 4. ¿Cómo sería la velocidad inicial de una cubeta que se deja caer desde la azotea de un edificio hasta la superficie de la tierra? Para esta pregunta el $71,79 \%$ de los estudiantes contestó correctamente la opción C, afirmando que era cero debido a que parte del reposo. Las opciones de respuesta A, B y D tienen porcentajes del 10,26\%, 15,38\% y $2,56 \%$ respectivamente, los estudiantes consideraron que la velocidad inicial es diferente de cero; en la opción $A$ se señala que el cuerpo alcanza un máximo de velocidad al ser soltado y luego continúa con velocidad constante, en la opción B el cuerpo aumenta la velocidad por la acción gravitatoria a medida que se acerca a la superficie de la tierra y en la opción D la velocidad aumenta en la mitad de su trayectoria. La opción E no fue considerada como respuesta por ningún estudiante.

Pregunta 5. Cuestiona el tiempo que tarda en llegar al suelo una moneda y una hoja de papel extendida cuando se dejan caer desde la misma altura y en el mismo instante. Un porcentaje del 28,21\% de los estudiantes contestó correctamente la opción B, afirmando que el tiempo era menor para la moneda comparada con la hoja de papel extendida. La opción A fue la opción incorrecta más popular entre los estudiantes con el 38,46\%, considerando que el tiempo de la moneda era mayor que la hoja de papel extendida, seguida de la opción C con un $25,64 \%$, en la que se mencionaba que el tiempo era igual para ambos objetos. Las opciones D y E muestran porcentajes bajos de $2,56 \%$ y $5,18 \%$ respectivamente, en donde el tiempo es aproximadamente la mitad o la cuarta parte para la moneda comparada con la hoja de papel extendida. En relación con esta pregunta, es importante mencionar que luego de la aplicación de la prueba y al hacer un conversatorio con los estudiantes, se puede 
considerar modificar la pregunta en la prueba o reformularla, haciendo énfasis en la situación en ausencia de aire y considerando su resistencia. Dado que como está redactada generó algunas dudas porque no es claro si se considera o no el efecto del rozamiento del aire sobre la hoja de papel. Posiblemente esto hizo que se obtuvieran los resultados que se presentan.

Ítem 6. La situación planteada indaga sobre la aceleración de una pelota de pingpong que se deja caer desde el techo de un edificio de dos pisos hasta la superficie de la tierra. En esta pregunta, el 56,41\% de los estudiantes contestó correctamente al elegir la opción $A$, admitiendo que la aceleración es constante. Se puede apreciar que la respuesta B tiene un porcentaje del 23,08\%, los estudiantes consideraron que la aceleración aumentaba debido a la atracción gravitatoria que se hace mayor a medida que el objeto se acerca a la superficie. Las opciones $C$ y D muestran porcentajes inferiores al 12,82\%, parece que no se llega a comprender que la aceleración es constante en cualquier punto de la trayectoria, la opción E no fue considerada como respuesta entre los estudiantes.

Numeral 7. Se cuestiona la aceleración de una moneda que se deja caer desde dos alturas distintas $(\mathrm{H}$ y h) considerando que la altura $\mathrm{H}$ es exactamente el doble de la altura h. Se observa que el 43,59\% de los estudiantes contestó correctamente (opción C), afirmando que la aceleración es igual en ambos casos. La opción A, aunque es una respuesta incorrecta, fue seleccionada por el 38,46\% de los estudiantes, quienes consideran que la aceleración era mayor para la altura $\mathrm{H}$. Las opciones $\mathrm{B}, \mathrm{D}$ y E son poco consideradas por los estudiantes, logrando porcentajes inferiores al 10,26\%.

Numeral 8. ¿Cómo sería el tiempo para la moneda si se deja caer de dos alturas $(\mathrm{H}$ y h) teniendo en cuenta que la altura $\mathrm{H}$ es exactamente el doble de la altura h? Los resultados muestran que un $82,05 \%$ de los estudiantes contestó correctamente la pregunta con la opción D, afirmando que este era mayor si cae de la altura $\mathrm{H}$. Para las otras opciones, $A, B$ y $C$, se tienen porcentajes inferiores al $7,69 \%$. La opción $E$ no fue considerada por ningún estudiante.

Interrogante 9. ¿Cómo sería la velocidad para la moneda si se deja caer de dos alturas $(\mathrm{H}$ y $\mathrm{h}$ ) teniendo en cuenta que la altura $\mathrm{H}$ es exactamente el doble de la altura h? En esta pregunta, un 61,54\% de los estudiantes contestó correctamente al elegir la opción A, considerando que la velocidad adquiere un valor distinto en cada punto de su trayectoria. La respuesta $B$ fue seleccionada con un porcentaje del 20,51\%, afirmando que la velocidad es constante. Las opciones C, D y $E$ muestran porcentajes bajos del 5,13\%, $10,26 \%$ y $2,56 \%$, la opción E no fue seleccionada por ningún estudiante.

En la siguiente figura se relacionan los resultados correctos por pregunta, para el pretest y postest; en la tabla 4 se presenta el índice o factor de Hake (ganancia de aprendizaje global).

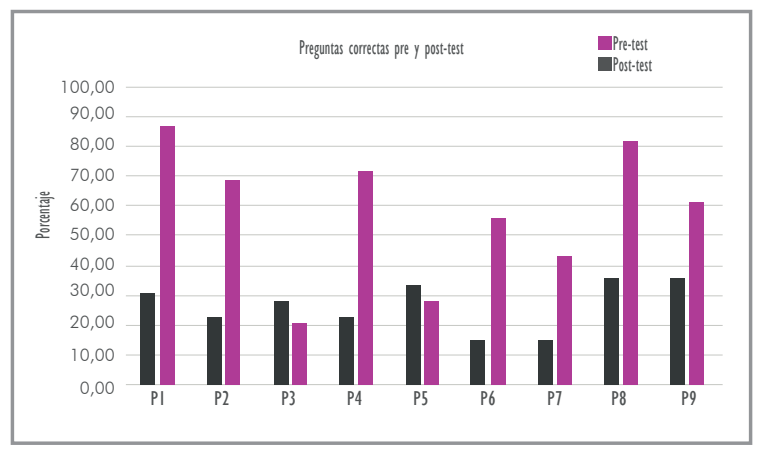

Figura 10. Porcentaje de preguntas correctas pre y postest

Fuente: elaboración propia. 
Tabla 4. Coeficiente Hake global del grado décimo

\begin{tabular}{|c|c|c|c|}
\hline Grado & Pretest (\%) & Postest (\%) & $\begin{array}{c}\text { Índice de } \\
\text { Hake }\end{array}$ \\
\hline Décimo & 26,78 & 57,83 & 0,42 \\
\hline
\end{tabular}

Fuente: resultados prueba pre y postest aplicada a los estudiantes de grado 10-1 de la Institución Educativa Emiliano Restrepo.

La ganancia de aprendizaje, según el índice de Hake, se clasifica en el rango medio entre 0,3 y 0,7 . Se observa un avance significativo en la comprensión del movimiento en caída libre.

\section{Resultado por grupo de preguntas y su análisis}

Para una mejor comprensión, se clasificaron las preguntas en tres grupos o variables, atendiendo a los conceptos que evalúa. La agrupación de preguntas se ilustra en la tabla 5.

Tabla 5. Agrupación de preguntas

\begin{tabular}{|c|c|}
\hline Variable & Agrupación de preguntas \\
\hline Tiempo de caída & P1, P5, P8 \\
\hline Velocidad & P2, P4, P9 \\
\hline Aceleración & P3, P6, P7 \\
\hline
\end{tabular}

Fuente: Riveros (2016).

La figura 11 presenta el resultado del pretest y postest por grupos de preguntas según las categorías dadas en la tabla 5. En la tabla 6 se presenta el índice o factor de Hake asociado a dichas variables.

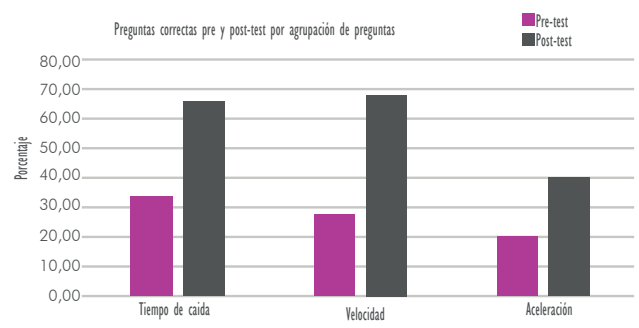

Figura 11. Promedio de preguntas correctas pre y postest por agrupación de preguntas Fuente: resultados prueba pre y postest aplicados a los estudiantes de grado 10-1 Institución Educativa Emiliano Restrepo. 
Tabla 6. Coeficiente o factor de Hake por grupo de preguntas

\begin{tabular}{|c|c|c|c|}
\hline Variable & Pretest (\%) & Postest (\%) & $\begin{array}{c}\text { Índice de } \\
\text { Hake }\end{array}$ \\
\hline $\begin{array}{c}\text { Tiempo de } \\
\text { caída }\end{array}$ & 33,33 & 65,81 & 0,49 \\
\hline Velocidad & 27,35 & 67,52 & 0,55 \\
\hline Aceleración & 19,66 & 40,17 & 0,26 \\
\hline
\end{tabular}

Fuente: resultados prueba pre y postest aplicada a los estudiantes de grado 10-1 de la Institución Educativa Emiliano Restrepo.

De estas figuras y tablas se puede concluir que en cuanto al tiempo de caída se tuvo un buen nivel de asimilación (rango medio de ganancia de aprendizaje), que se puede atribuir de las situaciones físicas ejecutadas durante la unidad didáctica, así como la discusión de resultados y contrastación de predicciones; para la variable de velocidad se presentó una ganancia relativa de aprendizaje media. En relación con la aceleración, la ganancia relativa de aprendizaje es baja, los estudiantes tienen dificultades para asociar que en el movimiento de caída libre o lanzamiento vertical hacia arriba el cambio de la velocidad en función del tiempo es proporcional y que este hecho está relacionado con la aceleración constante. Se hace necesario implementar más situaciones experimentales que posibiliten mayor apropiación conceptual, en donde se analicen las variables físicas que intervienen; por ejemplo, disponer de esferas de poliestireno del mismo tamaño, hacerles hueco y rellenarlas de diferentes materiales, de tal forma que queden unas más pesadas que otras, dejar caer dos a dos las esferas, indagar a los alumnos por cuál llegará primero al suelo y por qué, realizar la práctica y discutir los resultados, repetir la situación, haciendo uso de celulares para grabar la caída de los cuerpos, y con ayuda de videotracker realizar el análisis de variables, enfatizando en la aceleración del movimiento.

\section{Consideraciones finales}

La UD diseñada bajo la MAA contribuyó en el mejoramiento de los procesos de enseñanza-aprendizaje de la física, toda vez que los estudiantes abordaron los conceptos básicos a partir de su propia experiencia. También se fomentaron espacios de discusión que les permitieron a los estudiantes contrastar sus ideas previas con los resultados de la práctica.

De acuerdo con la clasificación propuesta por Richard Hake al analizar los resultados de la ganancia normalizada promedio, después de las intervenciones con el instrumento de diagnóstico (pretest y postest), se encuentra que los estudiantes se ubican en una zona de ganancia media $(0,42)$, logrando un avance conceptual; los resultados se pueden explicar a partir de las relaciones que hacen los alumnos de las variables que intervienen en el movimiento, a saber: tiempo de caída, velocidad y aceleración. En este sentido, en la comprensión de los conceptos de tiempo de caída y velocidad hubo un avance significativo, pero en el concepto de aceleración no hubo mayor ganancia conceptual, dado que luego de las intervenciones continúan afirmando que a medida que el cuerpo cae gana aceleración, por tanto en el diseño de la UD, se deben incluir más experiencias que les permitan a los estudiantes hacer el análisis de la variación de la velocidad para un cuerpo en caída libre, o en movimiento vertical hacia arriba y su relación con la aceleración.

Es deseable que se continúe trabajando con los estudiantes en propuestas alternativas como la discutida en este artículo para la enseñanza de la física, de tal forma que se afiancen los conceptos propuestos en la unidad didáctica y se planteen más experiencias que le permitan al estudiante la descripción, no solo cualitativa de la situación, sino la medición de variables y la verificación de hipótesis mediante el análisis cuantitativo de la experiencia. 
Gracias al trabajo descrito se comprobó que los estudiantes presentaron una mejor apropiación conceptual, trabajaron en equipo de manera asertiva, y modificaron su interés, motivación y valoración de las ciencias. Esta metodología de aprendizaje busca una participación activa por parte del estudiante, brindándole la oportunidad de redescubrir la ciencia y cambiar la percepción que tiene de ella.

La elaboración de unidades didácticas usando la metodología presentada le permite al docente estructurar de manera secuencial y articulada cualquier situación particular.

\section{Agradecimientos}

A la Universidad de los Llanos por el apoyo financiero en la ejecución del proyecto Diseño e implementación de la metodología de aprendizaje activo para la enseñanza de física de grado décimo, C03-F03-38-2015.

A la Institución Educativa Emiliano Restrepo Echavarría del municipio de Restrepo (Meta).

\section{Referencias}

Barbosa, L. H. (2008). Los experimentos discrepantes en el aprendizaje activo de la física. Latin-American Journal of Physics Education, 2 (3), 246-252.

Barrera, K. J. (2007). La enseñanza de la física a través de habilidades investigativas: una experiencia. Latin-American Journal of Physics Education, 1 (1), 39-43.

Benegas, J. (2007) Tutoriales para física introductoria: una experiencia exitosa de aprendizaje activo de la física. Latin-American Journal of Physics Education, 1(1), 32-38.

Benegas, J., Sokoloff, D. R, Priscilla, L., Zavala, G., Punte, G., Gangoso, Z. y Alarcón, H. (2011). Manual de entrenamiento. 4. ${ }^{\circ}$ Taller regional del Cono Sur sobre Aprendizaje Activo de Física: Termodinámica y Fluidos (AATyF-Córdoba). Recuperado de http://www.famaf.unc.edu.ar/congresos/taaf/index.html

Bruner, J. (1987). La importancia de la educación. Barcelona: Paidós Educador.

Campanario, J. M. y Moya, A. (1999). ¿̇Cómo enseñar ciencias? Principales tendencias y propuestas. Enseñanza de las Ciencias, 17(2), 179-192.

Elizondo, M. S. (2013). Dificultades en el proceso de enseñanza aprendizaje de la física. Presencia Universitaria, 3 (5), 70-77.

Franco, R., Velasco, M. y Riveros, C. (2017). Los trabajos prácticos de laboratorio en la enseñanza de las ciencias: tendencias en revistas especializadas: 2012-2016. Tecné, Episteme y Didaxis, 41, 37-56. 
Garduño, L. (2010). Implementación de clases demostrativas interactivas para la enseñanza de caída libre en el bachillerato. (Tesis de Maestría en Ciencias en Física Educativa). Instituto Politécnico Nacional-Centro de Investigación en Ciencia Aplicada y Tecnología Avanzada, México.

Garduño, L., López, A., Mora C. (2013). Evaluación del aprendizaje conceptual del movimiento en caída libre. Latin-American Journal Physics Education, 7(2), 275-283. Recuperado de: http://www.lajpe.org/jun 13/ LAJPE_777_Lilia_GarduA_o_preprint.pdf

Gil, D. y Valdés, P. (1996). La orientación de las prácticas de laboratorio como investigación: un ejemplo ilustrativo. Enseñanza de las Ciencias, 14 (2), 155-163.

Hake, R. R. (1998). Interactive-engagement versus traditional methods: A six-thousand-student survey of mechanics test data for introductory physics courses. American Journal of Physics, 66(1), 64-74.

Hestenes, D., Wells, M. y Swackhamer, G. (1992). Force concept inventory. The Physics Teacher, 30(3), 141-158

McDermott, L. C. (2001). Oersted medal lecture 2001: "Physics education research—the key to student learning". American Journal of Physics, 69(11), 1127-1137.

McDermott, L.C., (1993) Guest comment: How we teach and how students learn: $A$ mismatch. American Journal of Physics, 61, 295-298.
Silberman, M. (1998). Conducting an active training program. Active Training, 207-289.

Solbes, J., Monserrat, R. y Furio, C. (1997). El desinterés del alumnado hacia el aprendizaje de la ciencia: implicaciones en su enseñanza. Didáctica de las Ciencias Experimentales y Sociales. 21, 91-117.

Sokoloff, D. R., y Thornton, R. K. (2004). Interactive lecture demonstrations. Interactive Lecture Demonstrations. Wiley-VCH.

Thornton, R. K. y Sokoloff, D. (1997). Using interactive lecture demonstration to create an active learning environment. The Physics Teacher 35, 340-347.

Villarreal, M., Lobo, H., Gutiérrez, G., Briceño, J., Rosario J., Díaz, J. C. (2005). La enseñanza de la física frente al nuevo milenio. Universidad de los Andes-Venezuela, Núcleo Universitario Rafael Rangel. Revista Academia.

Zapata, J., Moreno, N., Badillo, R., Rosero, S., Flórez, Y. y Rodríguez, L. (2012). Enseñanza del magnetismo en la formación inicial de profesores un abordaje desde el aprendizaje activo. Revista Virtual educyt, 15.

\section{Para citar este artículo:}

Martínez Velásquez, N. Y. y Riveros Míguez, S. Y. (2018). La enseñanza de caída libre bajo la metodología de aprendizaje activo. Tecné, Episteme y Didaxis, 45, 35-51. 


\section{Anexo 1}

Prueba diagnóstica o de entrada

Prueba de entrada

Nombre:

Grado:

Fecha:

Instrucciones: a continuación, encontrará nueve preguntas ${ }^{3}$. Debe responderlas de forma ordenada en la hoja de respuestas dispuesta para tal fin, argumentando en cada caso la opción que escoja, si es preciso acompañe las respuestas de gráficos y esquemas. Tan pronto termine entregue la prueba al docente junto con la hoja de respuestas. Trabaje de forma individual y analice muy bien cada pregunta.

1. Dos esferas de metal tienen el mismo tamaño, pero una pesa el doble que la otra. Se dejan caer estas esferas desde el techo de un edificio de un solo piso en el mismo instante de tiempo. El tiempo que tardan las esferas en llegar al suelo es:

A. Aproximadamente la mitad para la esfera más pesada que para la esfera más liviana.

(B). Aproximadamente la mitad para la esfera más liviana que para la esfera más pesada.

C. Aproximadamente el mismo para ambas.

D. Considerablemente menor para la esfera más pesada, pero no necesariamente la mitad.

E.). Considerablemente menor para la esfera más liviana, pero no necesariamente la mitad.

6. Las dos bolas de metal del problema anterior ruedan sobre una mesa horizontal con la misma velocidad y caen al suelo al llegar al borde de la mesa. En esta situación:

A. Ambas bolas golpean el suelo aproximadamente a la misma distancia horizontal de la base de la mesa.

(B). La bola más pesada golpea el suelo aproximadamente a la mitad de la distancia horizontal de la base de la mesa que la bola más liviana.

C. La bola más liviana golpea el suelo aproximadamente a la mitad de la distancia horizontal de la base de la mesa que la bola más pesada.

3 Garduño Calderón Lilia. 2010. Implementación de las clases demostrativas interactivas para la enseñanza de caída libre en el bachillerato. (Tesis de Maestría en Ciencias en Física Educativa). Instituto Politécnico Nacional. Centro de Investigación en Ciencia Aplicada y Tecnología Avanzada. 
D. La bola más pesada golpea el suelo considerablemente más cerca de la base de la mesa que la bola más liviana, pero no necesariamente a la mitad de la distancia horizontal.

E. La bola más liviana golpea el suelo considerablemente más cerca de la base de la mesa que la bola más pesada, pero no necesariamente a la mitad de la distancia horizontal.

6. Una piedra que se deja caer desde el techo de un edificio de un solo piso hasta la superficie de la tierra:

A. Alcanza un máximo de velocidad muy pronto después de ser soltada y desde entonces cae con una velocidad constante.

(B). Aumenta su velocidad mientras cae porque la atracción gravitatoria se hace considerablemente mayor cuanto más se acerca la piedra a la tierra.

C. Aumenta su velocidad porque una fuerza de gravedad casi constante actúa sobre ella.

D. Cae debido a la tendencia natural de todos los objetos a descansar sobre la superficie de la tierra.

E. Cae debido a los efectos combinados de la fuerza de la gravedad, empujándola hacia abajo, y la fuerza del aire, también empujándola hacia abajo.

6. Una cubeta se deja caer desde la azotea de un edificio hasta la superficie de la tierra, su velocidad inicial:

A. Alcanza un máximo de velocidad al ser soltada y desde entonces cae con una velocidad constante.

(B). Aumenta su velocidad al ser soltada porque la atracción gravitatoria se hace considerablemente mayor cuanto más se acerca a la tierra.

C. Es cero, ya que parte del reposo.

D. Aumenta aproximadamente a la mitad de su trayectoria.

(E). Disminuye aproximadamente a la mitad de su trayectoria.

6. Una moneda y una hoja de papel extendida se dejan caer desde la misma altura y en el mismo instante. El tiempo que tardan en llegar al suelo es:

A. Mayor para la moneda comparada con la hoja de papel extendida.

(B). Menor para la moneda comparada con la hoja de papel extendida.

C. Igual para los dos cuerpos. 
D. Aproximadamente la mitad para la moneda comparada con la hoja de papel extendida.

E.). Aproximadamente la cuarta parte para la moneda comparada con la hoja de papel extendida.

6. Una pelota de ping-pong que se deja caer desde el techo de un edificio de dos pisos hasta la superficie de la tierra:

A. Al ser soltada mantiene su aceleración casi constante.

(B). Aumenta su aceleración mientras cae porque la atracción gravitatoria se hace considerablemente mayor cuanto más se acerca la piedra a la tierra.

C. Aumenta su aceleración porque una fuerza de gravedad casi constante actúa sobre ella.

D. Disminuye su aceleración debido a la tendencia natural de todos los objetos al caer.

E.). Disminuye su aceleración debido a que la fuerza de gravedad actúa sobre ella.

- Use la descripción y la figura adjuntas para contestar las siguientes cuatro preguntas (7 a 9)

Una moneda se deja verticalmente hacia abajo desde dos alturas distintas $(\mathrm{H}$ y h). Considerando que la altura $\mathrm{H}$ es exactamente el doble de la altura $h$.

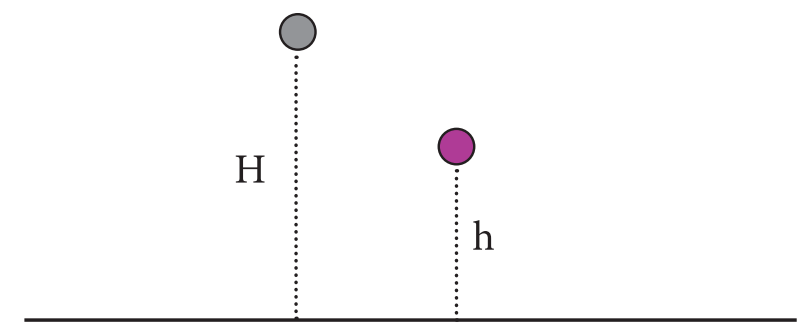

7. ¿Cómo es la aceleración de la moneda al final de su recorrido?

A. Mayor al caer de la altura $\mathrm{H}$.

(B). Menor al caer de la altura $\mathrm{H}$.

C. Igual en las alturas.

(D). Justamente la mitad de la altura $\mathrm{H}$, en la altura $h$.

54

E.) Ninguna de las anteriores. 
8. ¿Cómo es el tiempo que tarda la moneda en llegar al piso?

(A). Mayor si cae de la altura h.

(B). Menor si cae de la altura $\mathrm{H}$.

C. Igual en la altura $\mathrm{H}$ que en la altura h.

D. Mayor si cae de la altura $\mathrm{H}$.

E. Ninguna de las anteriores.

9. Cuándo la moneda se deja caer, su velocidad:

(A). Adquiere distinto valor en cada punto de su trayectoria.

(B). Presenta el mismo valor en cada punto de su trayectoria.

C. Se comporta inversamente proporcional a su aceleración.

D. Es igual a su aceleración.

(E). Ninguna de las anteriores.

\section{Hoja de respuestas}

Marque con una " $x$ " la opción que considere correcta en cada caso. No marque más de dos opciones por pregunta, si hace esto la pregunta se anula.

\begin{tabular}{|c|c|c|c|c|c|c|c|c|}
\hline 1 & 2 & 3 & 4 & 5 & 6 & 7 & 8 & 9 \\
\hline (A) & (A) & (A) & (A) & (A) & (A) & (A) & (A) & (A) \\
\hline (B) & (B) & (B) & (B) & (B) & (B) & (B) & (B) & (B) \\
\hline (c) & (C) & (C) & (c) & (c) & (C) & (C) & (C) & (C) \\
\hline (D) & (D) & (D) & (D) & (D) & (D) & (D) & (D) & (D) \\
\hline (E) & (E) & (E) & (E) & (E) & (E) & (E) & (E) & (E) \\
\hline
\end{tabular}


